\title{
Edge Detection and Identification for Tooth Saw Butt Joint
}

\author{
Hairol Nizam Mohd Shah ${ }^{1}$, Muhammad Shadiq Lagani ${ }^{2}$, Zalina Kamis ${ }^{3}$, Mohd Rizuan Baharon ${ }^{4}$ \\ ${ }^{1,2,3}$ Centre for Robotics and Industrial Automation (CeRIA), Faculty of Electrical Engineering, Universiti \\ Teknikal Malaysia Melaka (UTeM), Hang Tuah Jaya, 76100 Durian Tunggal, Melaka, Malaysia, \\ hnizam@utem.edu.my. \\ ${ }^{4}$ Department of Computer System and Communication, Faculty of Communication and Information Technology, \\ Universiti Teknikal Malaysia Melaka (UTeM), Malaysia.
}

\begin{abstract}
Nowadays, the most well-known autonomous method in recognizing and identifying the edge of a butt joint implements the usage of vision sensor or laser-assisted vision sensor because of its performance and robustness compared to a manual approach. This research paper presents the development of a vision-based method to recognize and identify the edge of a tooth saw butt joint shape. The methodology to recognition and identification edge for tooth saw butt joint shape comprises into four processes: (1) image pre-processing (2) image segmentation (3) morphological (4) edge butt joint feature points representation and description. The edge points for tooth saw butt joint shape that is start point, support point $1 \& 2$ and the end point represented in $\mathrm{x}$ and y coordinates. The edge recognition and identification for tooth saw butt joint shape was obtain using different edge detection techniques such as Sobel, Prewitt, Roberts and Canny edge detection techniques. The repeatability is based on the standard deviation obtain from the average points observed from edge detection techniques. The results show that Canny edge detection technique is the most accurate method compare the others while all the techniques has a high repeatability due to the reliability of the variables obtained
\end{abstract}

Key words : edge detection and identification, tooth saw, features point, morphological

\section{INTRODUCTION}

Today's current method in recognizing and identifying the edge of a tooth saw butt joint shape in manufacturing processes, mainly in modern robotic welding are using manual operation which is by depending on human observations. The robot is customized and calibrated using "teach and playback" techniques by skilled human operators each time a new edge of tooth saw butt joint shape with different measurements is introduced. Through this repetitive process, it can be time consuming causing the rate of production of the industry using this current method to have a low rate of production. The complexity shape of the edge of a tooth saw butt joint also contribute in the time consumption of operation. It also increases cost to hire several skilled operators to operate the robot manually $[1,2,3]$.

In order to solve the problem, a fundamental machine vision engineering knowledge is required. A vision-based system with good illumination source can be applied to the robotic welding enabling it to recognize and identify the feature points (start, supporting and end) of the edge of the tooth saw butt joint shape automatically. By doing so, the duration of operation in recognition of the edge of the tooth saw butt joint shape will be brief. The implementation of the vision-based system will also reduce the cost required to hire many skilled operators. Digital image processing techniques is required to process the image captured by the vision-based system of the edge of a tooth saw butt joint shape which enables the feature points of the edge of a tooth saw to be extracted. The techniques consist of enhancement, segmentation, edge detection and morphological operation [4]. Hence, through those techniques, the feature points of the edge of a tooth saw butt joint shape can be described and presented.

\section{LITERATURE REVIEW}

HNM Shah et. al [1] developed vision sensor from a CCD camera with $533 \times 400$ pixel size. By using local thresholding method, a problem based on variation of lighting condition, shadow and material reflection to recognize straight, curve and tooth saw shapes seam path can be reduce. On the other hand, M. Dinham [2] introduced a stereo matching algorithm to identify the weld seam shapes, which are straight, curve and tooth saw shape used CCD stereo (dual) camera with $1280 \times 1024$ pixel size. The image are captured using two cameras, left and right. The left image is use for extraction of weld seam line and the right image is for matching feature points calculation.

Y. He and team [3] used a laser assisted vision sensor, CCD camera with $768 \times 576$ pixel size using method of automatic 
multi-pass planning, by applying the polynomial fitting plus derivatives to extract the feature points of the weld seam profile of the butt joint. Meanwhile, D. Kaur et. al [5] also developed a laser assisted vision sensor, but used CMOS as the camera with 1024 x 1280 pixel size. The system implemented according to target tracking method based on Gaussian kernel to extract the butt, lap and curve weld shapes. An adaptive fuzzy controller was design to give an input to deviation value of the feature points and the change rate of the deviation into the controller.

Y. Zou et. al [6] developed a vision sensor by implemented a CCD camera. The system using a novel software program includes specific modules, welding power control, intelligent parameter setting, image sensing algorithm, welding database robot communication and path planning modules to extract the feature points of the straight weld seams. In the meantime, Y. Xu et. al [7] implemented a laser assisted sensor by using CCD camera with $7.4 \mu \mathrm{m}$ pixel size. A three laser stripes with different wavelength which is projected on the weld seam, two red laser strips are used to measure the three dimensional profile of the weld groove. Then the optical triangulation and the third green laser strip is apply as light source to measure the edge of the $\mathrm{V}$, square, closed square grooves.

W.J. Shao and team [8] developed weld seam detection for space weld of narrow butt joint in laser welding. The system used a traditional morphological method with aid from the laser assisted vision sensor to recognize the weld seam shape of a $\mathrm{C}$ and $\mathrm{S}$ butt joint shape. Meanwhile, authors in [9] implemented seam-tracking system based on a new structured light vision sensor with optical filters and an extra LED light to filter out most of the noises from strong arc lights. An image processing is design to obtain the feature points of the straight butt seams both in horizontal and vertical directions. Two independent fuzzy-PID controllers are used to achieve seam-tracking control accurately.

Authors in [10], used a vision sensor with a manipulation of a directional lighting and CCD camera with $1600 \times 1200$ pixel size. By using two directional lights projected onto the edges of the straight seam shape edge, features can be obtain from the distinct man-made "light and shadow" features. Meanwhile, J. Zeng [11] developed a visual weld edge recognition based on light and shadow features. A circular laser trajectory can be determined weld seam location and seam tracking. The identification and detection of initial, mid and end points of weld seam by using Harris binomial, Harris, Lepetit and Sojka points detector introduced by HNM Shah et al [12]. A CCD camera which is placed in an ideal position to generate the information of $\mathrm{x}$ and $\mathrm{y}$ coordinates.

Hence, based on the literature reviews on the theories background and related past researches, there are four main steps to recognize and identify the tooth saw butt joint shape. The steps are; (1) image pre-processing (2) image segmentation (edge detection technique) (3) morphological image processing (dilation and erosion) and (4) feature points description and representation (skeletonization and corner points detection). In order to do so, the equipment needed are vision sensor, CMOS camera, illumination source and an image processing software.

\section{METHODOLOGY}

\subsection{Variables Validations}

In an image processing process, variables such as the illumination brightness can determined a good quality image. The threshold value for edge detection techniques will be give an information on the edges of the tooth saw butt joint shape, structuring element size for morphological operation which is dilation to link and combine unlinked.

\subsection{Validation of Reference Points}

The goal of the references point is to identify the original feature points of the edge of the tooth saw butt joint shape. The identified points are in pixel coordinates value and denoted as start point, supporting point $1 \& 2$ and end point. This task also initializes the initial position of the region of interest ROI which will help in the image processing process onwards. Image of the workpiece containing the edge of the tooth saw butt joint shape is captured. From the captured image, the start point, supporting point $1 \& 2$ and end point is marked using human observation and the pixel coordinate for each marked point is recorded as the original point.

The start point is determined as the lowest column of the image and the end point is determined as the highest column of the image. The supporting points are determined from the intersection of the two lines produce by the start and end point with the middle line of the edge tooth saw butt joint. The points for region of interest is also determined. ROI 1 is used as a window which inside the window contains the edge of the tooth saw butt joint shape and ROI 2 is applied for the removal of unwanted edges in the image processing process [1]. Figure 1 shows the positions of each feature points that is marked.

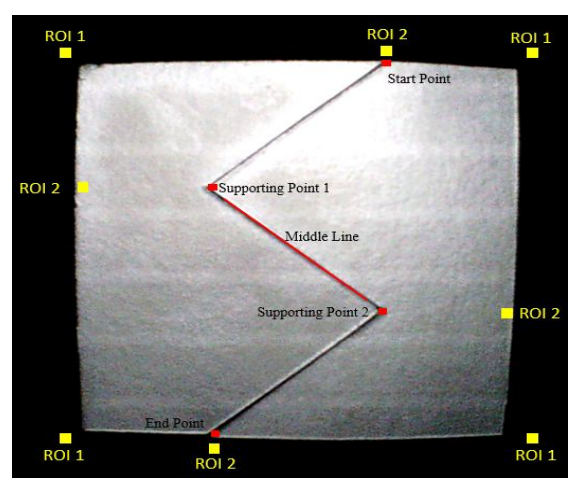

Figure 1: Position of marked points 


\subsection{Identification of Feature Points of Edge of the Tooth Saw Butt Joint Shape}

The general technique used in the edge detection is by using a gradient based edge detection such as, Canny, Prewitt, Sobel and Roberts edge detector. Figure 2 shows the flowchart of edge detection process.

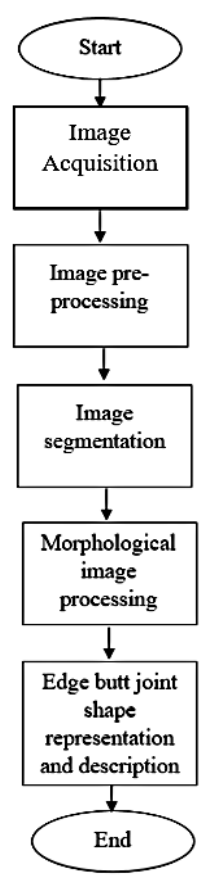

Figure 2: Edge detection process

\subsubsection{Image Acquisition}

The CMOS camera is control by a program coded in the MATLAB image processing toolbox to capture the image of the work piece containing the edge of the tooth saw butt joint shape using the function "snapshot". The acquired image is then used in further analysis. Figure 3 shows the original image that had been captured and filtered out using the ROI isolation to reduce unwanted information processed

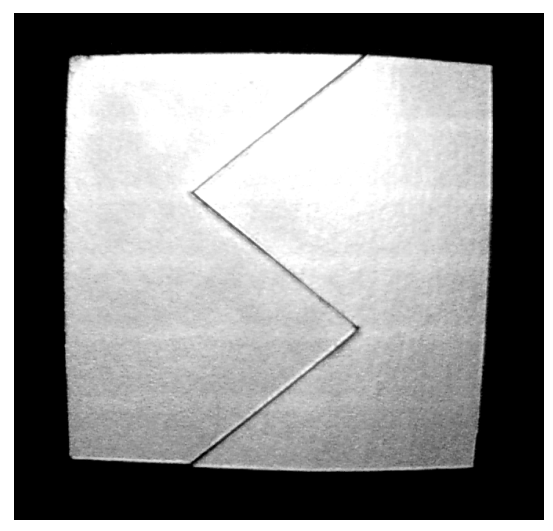

Figure 3: Original image

\subsubsection{Image Segmentation}

Edge detection method is use in order to detect the edge of the tooth saw butt joint shape. The edge detection method that is used is the gradient based edge detection which is by using Roberts, Sobel, Prewitt and Canny Operator. The original image captured is a function of two variables, $\operatorname{img}(\mathrm{i}, \mathrm{j})$ where img is the function of the original image which returns the intensity pixel value of the original image at point $(i, j)$. The point $(i, j)$ is the intensity value of each pixel in the original image, which is between 0 to 255 . Thus, the derivative for the function $\operatorname{img}(i, j)$ is depicted as Eq. (3) [5].

$$
\nabla i m g=i \frac{\operatorname{aing}\left(i_{j}\right)}{a t}+j \frac{\operatorname{aimg}\left(\phi_{j}\right)}{a j}
$$

where is the gradient for horizontal direction and is the gradient for vertical direction. The gradient magnitude, $|\mathrm{G}|$ of the original image can be depicted as Eq. (4) [15].

$$
|G|=\sqrt{\left(\frac{\operatorname{dim} g(i, j)}{\partial i}\right)^{2}+\left(\frac{\operatorname{dim} g(i, j)}{\partial j}\right)^{2}}
$$

\subsubsection{Roberts, Sobel and Prewitt Edge Detection}

There are 5 steps in implementation of edge detection algorithms. The steps are:

Step 1: The original image is pre-processed using filters in order to reduce noise.

Step 2:The original image is convoluted in the horizontal direction using the x-kernel gradient component, $D_{x}$ of each gradient based edge detection operation according to Eq. (5) (7) $[5]$.

$$
\begin{gathered}
D_{x}=\left[\begin{array}{cc}
1 & 0 \\
0 & -1
\end{array}\right], \text { Roberts } \\
D_{x}=\left[\begin{array}{lll}
-1 & 0 & +1 \\
-2 & 0 & +2 \\
-1 & 0 & +1
\end{array}\right], \text { Sobel } \\
D_{x}=\left[\begin{array}{lll}
-1 & 0 & +1 \\
-1 & 0 & +1 \\
-1 & 0 & +1
\end{array}\right], \text { Prewitt }
\end{gathered}
$$

Step 3: The original image is convoluted in the vertical direction using the y-kernel gradient component, $D_{y}$ of each gradient based edge detection operation according to Eq. (8) - (10) [5].

$$
D_{y}=\left[\begin{array}{cc}
0 & 1 \\
-1 & 0
\end{array}\right], \text { Robert }
$$




$$
\begin{aligned}
& D_{y}=\left[\begin{array}{ccc}
-1 & 0 & +1 \\
-2 & 0 & +2 \\
-1 & 0 & +1
\end{array}\right], \text { Sobel } \\
& D_{y}=\left[\begin{array}{ccc}
+1 & +1 & +1 \\
0 & 0 & 0 \\
-1 & -1 & -1
\end{array}\right] \text {. Prowitt }
\end{aligned}
$$

Step 4: The magnitude of the gradient components for each pixel in the original image is calculated using Eq. (11) and compared with a threshold that had been chosen from the validation of threshold value analysis. If the magnitude value calculated is greater than the threshold value, the pixel will be defined as an edge and converted as a binary image with value of 1 . If the magnitude value calculated is lower than the threshold value, the pixel will be defined as unnecessary pixel and converted as a binary image with value of 0 [13].

$$
|D|=\sqrt{D_{x}^{2}+D_{y}^{2}}
$$

Step 5: The steps from 1 to 4 is repeated for each of the pixel in the original image.

\subsubsection{Canny Edge Detection}

In Canny detection, the algorithm isolates the background noise from the image to find discontinuities boundary, which is the edge of the tooth saw butt joint shape in the image captured. Canny edge detection provides the most optimal solution to remove noise from image due to these steps [5]. The steps are:

Step 1: Gaussian filter is used to smoothen the image inside the region of interest, removing noise and unwanted information using Eq. (12) - (13) [13].

$$
\begin{gathered}
g(x, y)=G \sigma(i, j) * i m g(i, j) \\
G \sigma=\frac{1}{\sqrt{2 \pi \sigma^{2}}} e^{\left(-\frac{i^{\mathbb{D}}+j^{2}}{2 \sigma^{2}}\right)}
\end{gathered}
$$

where $G \sigma(i, j)$, is the Gaussian filter, img $(i, j)$ is the original image captured, $\mathrm{g}(\mathrm{x}, \mathrm{y})$ is the smoothed image and $\sigma$ is the standard deviation referring to the Gaussian filter.

Step 2: The edges from the original image captured is calculated by applying the gradient to $\mathrm{x}$ and $\mathrm{y}$ coordinates of the captured image using Eq. (14) [13].

$$
M(x, y)=\sqrt{g_{x}^{2}(x, y)+g_{y}^{2}(x, y)}
$$

where $M(x, y)$ is the gradient, $g_{x}(x, y)$ and $g_{y}(x, y)$ are $\mathrm{x}$ and y coordinates respectively.

Step 3: The edges of the captured image is traced by defining a threshold using the $\mathrm{x}$ and $\mathrm{y}$ coordinates using Eq. (15) [24].

$$
M_{T}(x, y)\left\{_{0}^{M(x, y) \text { if } M(x, y)>T}\right.
$$

where $M_{T}(x, y)$ is the threshold value determined from the analysis of the validation of threshold value. The threshold value will determine where it will keep the information of the edge of the tooth saw butt joint shape and remove most of the noise present.

Step 4: The non-maximum pixels of the edge of the tooth saw butt joint shape is suppressed to obtain from the threshold $M_{T}$. To suppress it, each of the non-zero threshold determines whether it is greater than its neighboring pixels along the gradient. If it is greater, the threshold will not change. If it is less, it is set to 0 .

Step 5: In this step, the result from step 4 will be threshold to obtain two other different threshold which will be set as $\tau_{1}$ and $\tau_{2}$ which is defined as the high level and is defined as the low level. By doing so, two binary image will be generated and defined as $\boldsymbol{T}_{1}$ and .

Step 6: The final step is to link the edges in in $T_{z}$. To do this, each pixel that is defined as the edge in in $T_{z}$ will be located and the edges segmented of its neighbor binary image $T_{1}$ will also be located to link between both of the binary image. Thus a continuous edge will be formed.

Figure 4 shows the image segmented using edge detection for each edge detection technique, Roberts, Sobel, Prewitt and Canny respectively. It shows the edge detection by each edge detection techniques.

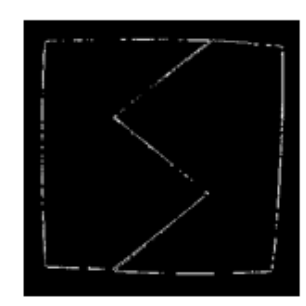

(a)

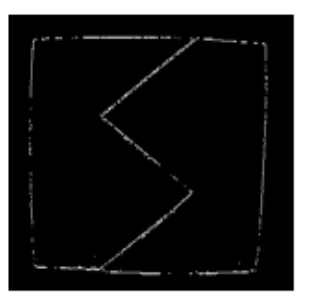

(c)

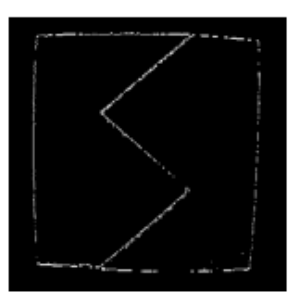

(b)

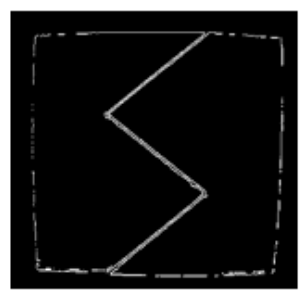

(d)
Figure 4: Edge detection techniques (a) Roberts (b) Sobel (c) Prewitt (d) Canny 


\subsubsection{Edge Butt Joint Shape Representation and Description}

This is the final process in order to obtain the feature points of the edge of the tooth saw butt joint shape. Skeleton technique is used onto the binary image after the morphological operation dilation step. This results in all the object inside the image converted to lines by thinning process, where it peels the contour of the edge of the tooth saw butt joint shape detected until it reaches most medial one-pixel width whilst preserving the topology of the edge shape [13].

Thus, the edge of tooth saw butt joint shape on the image is converted to a single line, which enables for the features points of the edge to be determined. The feature points are obtained by using Shi-Tomasi corner detection method. The method calculates a score which shows the variations of the pixel intensity in both $\mathrm{x}$ and $\mathrm{y}$ axes. The score is calculated as the minimum eigenvalues of the pixel intensity in both $\mathrm{x}$ and $y$ axes. Then a threshold is determined from the analysis of validation of minimum quality for corner detection. If the score is greater than the threshold determined, it will be defined as a corner. Eq. (17) shows the overview formula for the Shi-Tomasi corner detection method [15].

$$
R=\min \left(\lambda_{1}, \lambda_{2}\right)
$$

where $\mathrm{R}$ is the score $\lambda_{1}$ and $\lambda_{2}$ is the eigenvalues of the pixel intensity in both $\mathrm{x}$ and $\mathrm{y}$ axes.

\section{RESULTS}

\subsection{Experimental Setup}

A CMOS camera with a resolution of $1280 \times 720$ pixels size at 30 frames per second is use as the vision sensor to capture the workpiece image that contain the edge of the tooth saw butt joint shape. The camera is placed $20 \mathrm{~cm}$ vertically on top of the workpiece mounted on a customized stand. A black background workbench [16] is used to make the edge of the tooth saw butt joint shape more visible and ease the process of converting the original image to binary image.

The workpiece in this research is made from a hard cardboard material and spray painted with silver color to imitate the reflective behavior of an aluminum sheet. The workpiece dimension is $100 \mathrm{~mm} \times 100 \mathrm{~mm} \times 1 \mathrm{~mm}$, containing the tooth saw butt joint shape is placed under the camera on top of the black workbench in a fixed position to ensure a constant coordinates obtained from the image acquisition by the camera. This research is done in a control environment [17-18] where there is only one light source, which is the external LED light with a variable brightness between 50, 30 and 10 lumens. The LED light is positioned and adjusted until the edge of the tooth saw butt joint shape can be captured clearly by the CMOS camera. The detail of experimental setup is show in Figure 6.

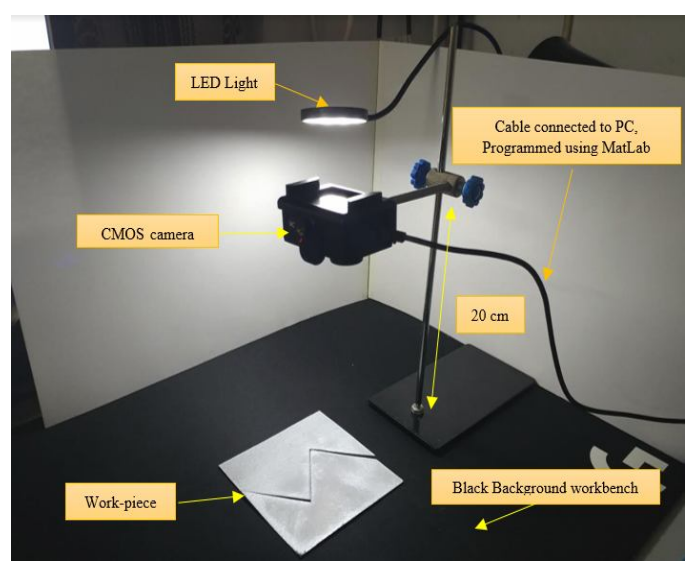

Figure 6: Experimental setup

\subsection{Validation of Reference Points Results}

Figure 7 shows the original image contain the edge of tooth saw butt joint shape. Each of the features point such as the starting point, supporting point $1 \& 2$ and end point is chosen and marked using human observation. Other important constant pixel points are also marked such as the ROI 1 and ROI 2 for the usage of image processing process.

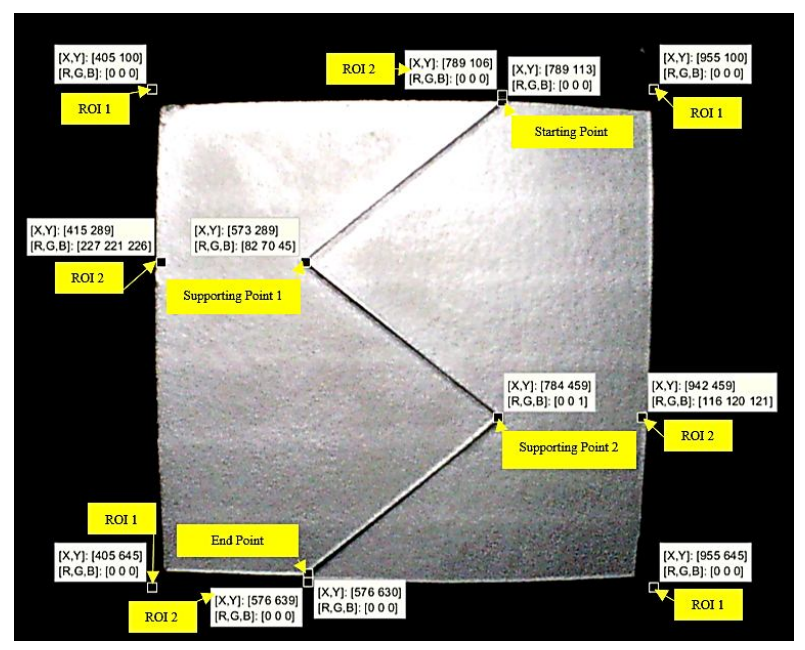

Figure 7: Marked Points

Table 1 and 2 shows the results for the preliminary task. The original start point, supporting point $1 \& 2$ and end point of the edge of the tooth saw butt joint shape obtained are in $\mathrm{x}$ and y coordinate pixel value. These points will act as the reference point.

Table 1: Original Points

\begin{tabular}{|l|l|}
\hline Point & Original Point (x, y pixel) \\
\hline Start & 789,113 \\
\hline Supporting 1 & 574,289 \\
\hline Supporting 2 & 784,459 \\
\hline End & 576,630 \\
\hline
\end{tabular}


Table 2: ROI Points

\begin{tabular}{|l|l|}
\hline Point & \multicolumn{1}{l}{$\begin{array}{l}\text { Average Identified Points (x, y } \\
\text { pixel) }\end{array}$} \\
\hline Roberts Edge Detector \\
\hline Start & $784.4,125.3$ \\
\hline Supporting 1 & $580.5,293.4$ \\
\hline Supporting 2 & $775.7,462.6$ \\
\hline End & $584.3,624.3$ \\
\hline Sobel Edge Detector & $783.4,125.3$ \\
\hline Start & $579.0,291.2$ \\
\hline Supporting 1 & $777.4,464.0$ \\
\hline Supporting 2 & $582.1,625.2$ \\
\hline End & $783.7,124.4$ \\
\hline Prewitt Edge Detector \\
\hline Start & $579.1,291.4$ \\
\hline Supporting 1 & $777.4,463.1$ \\
\hline Supporting 2 & $580.3,629.3$ \\
\hline End & $785.8,122.4$ \\
\hline Canny Edge Detector \\
\hline Start & $576.8,289.2$ \\
\hline Supporting 1 & $778.4,463.2$ \\
\hline Supporting 2 & $581.5,625.6$ \\
\hline End & \\
\hline
\end{tabular}

\subsection{Edge Butt Joint Shape Representation and Description}

The skeleton results towards the dilated image is show in Figure 8. The results show that the canny filter has the clear edge compare the others filters. shi-tomasi corner detection technique will be apply into the skeleton image to identify the feature points. This feature points also known as the corner of the tooth saw butt joint edge. Figure 9 show the Shi-Tomasi corner detection technique. Table 3 shows the average identification points taken from 10 times repetition process.

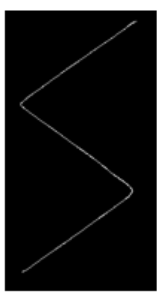

(a)

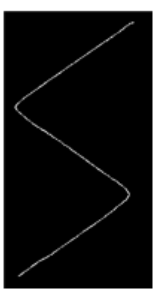

(b)

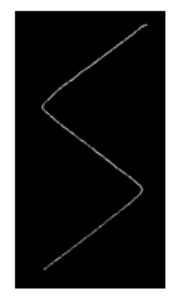

(c)

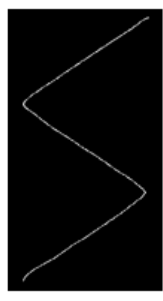

(d)
Figure 8: Skeletonization on (a) Roberts (b) Sobel (c) Prewitt (d) Canny

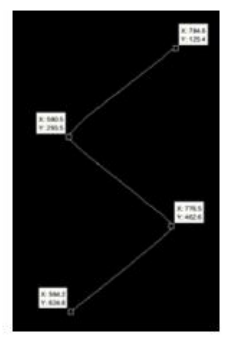

(a)

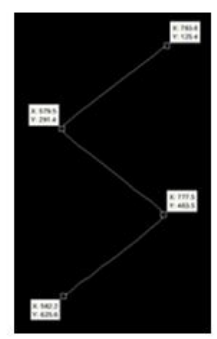

(b)

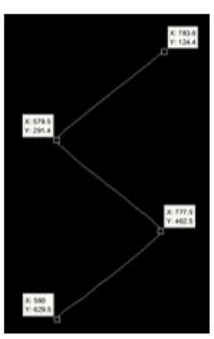

(c)

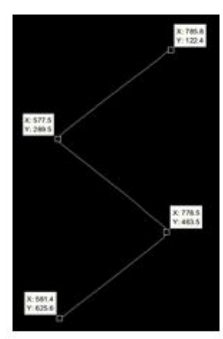

(d)
Figure 9: Corner detection on (a) Roberts (b) Sobel (c) Prewitt (d) Canny

Table 3: Identified Points

\begin{tabular}{|l|l|l|}
\hline Point & ROI 1 $(x, y$ pixel) & ROI 2 $(x, y$ pixel $)$ \\
\hline $\mathbf{1}$ & 405,100 & 789,106 \\
\hline $\mathbf{2}$ & 955,100 & 942,459 \\
\hline $\mathbf{3}$ & 955,645 & 576,639 \\
\hline $\mathbf{4}$ & 405,645 & 415,289 \\
\hline
\end{tabular}

\subsection{Accuracy Test Results}

Feature points of tooth saw butt joint edge using gradient-based edge detection (Canny, Prewitt, Sobel \& Roberts) is repeated for 10 times using the determined variables, which is the threshold value, structuring element size for the morphological operation dilation and the corner detection minimum quality. Each of the average result by using Eq. (18) of the repeated experiment is recorded.

$$
\bar{x}=\frac{\sum x_{n}}{n}
$$

where $\bar{X}$, is the average reading, $\sum x_{n}$ is the summation of each reading of the feature points detected and $n$ is the number of reading obtained.

The average coordinates of the feature points identified using each of the edge detection techniques are compared with the original point obtained from the validation of reference point. The accuracy is analyzed by determining which method identifies its features points near to the original reference points using Eq. (19).

$$
\Delta(\mathrm{x}, \mathrm{y})=\mathrm{x}(\mathrm{x}, \mathrm{y} \text { pixel })-\mathrm{y}(\mathrm{x}, \mathrm{y} \text { pixel })(\text { Mean }, \bar{x})
$$

where $\mathrm{x}$ is the original point and $\mathrm{y}$ is the average points identified using the proposed method

Table 5 shows the comparison error of each edge detection techniques for Canny, Prewitt, Sobel and Roberts edge detection technique to the original validated points. 
Hairol Nizam Mohd Shah et al., International Journal of Emerging Trends in Engineering Research, 8(8), August 2020, 4750 - 4757

Table 5: Accuracy test

\begin{tabular}{|l|l|}
\hline Point & Error $(\Delta \mathbf{x}, \Delta \mathbf{y})$ \\
\hline \multicolumn{2}{|l|}{ Roberts edge detection accuracy test } \\
\hline Start & $4.6,-12.3$ \\
\hline Supporting 1 & $-6.5,-4.4$ \\
\hline Supporting 2 & $8.3,3.6$ \\
\hline End & $-8.3,5.7$ \\
\hline Sobel edge detection & accuracy test \\
\hline Start & $5.6,-12.3$ \\
\hline Supporting 1 & $-5.0,-2.2$ \\
\hline Supporting 2 & $6.6,-5.0$ \\
\hline End & $-6.1,4.8$ \\
\hline Prewitt edge detection accuracy test \\
\hline Start & $5.3,-11.4$ \\
\hline Supporting 1 & $-5.1,-2.4$ \\
\hline Supporting 2 & $6.6,-4.1$ \\
\hline End & $-4.3,0.7$ \\
\hline Canny edge detection accuracy test \\
\hline Start & $3.2,-9.4$ \\
\hline Supporting 1 & $-2.8,-0.2$ \\
\hline Supporting 2 & $5.6,-4.2$ \\
\hline End & $-5.5,4.4$ \\
\hline
\end{tabular}

Based on Table 5 , it shows that the overall error value for Canny edge detector in the identification of the start point, supporting point $1 \& 2$ and the end point pixel coordinates is the least compared to the other three edge detection techniques. This is mean the Canny edge detector has a high accuracy in detecting edges. This is due to the hysteresis threshold where two thresholds that is pre-determined, which add more definition to the edge detection. Next, Prewitt edge detector shows a low value of error compared to Sobel and Roberts edge detection techniques even though three of those techniques use similar method where one threshold is predetermined in order to detect the edges. This is because the kernel used in Prewitt has better performance in determining the magnitude of the gradient in the image that contains the edges, compared to Sobel and Roberts edge detectors. Therefore, among the three edge detection techniques, Canny edge detector has a highest accuracy in finding important edges.

\subsection{Repeatability Test}

The average points from skeleton results is used to calculated the standard deviation. Low standard deviation value indicates that the method has less variation in the readings, thus high repeatability and reliability of the methods, procedures and variables used. Eq. (20) shows the formula to calculate standard deviation

$$
s=\sqrt{\frac{\sum\left(x_{n n}-x\right)^{2}}{n-1}}
$$

where is the value of each reading, is the average reading and is the number of reading obtained.
Table 6 shows the standard deviation of each edge detection technique, Canny, Prewitt, Sobel and Roberts Edge detector.

Table 6: Repeatability test

\begin{tabular}{|l|l|l|}
\hline Point & \multicolumn{1}{|c|}{$\begin{array}{l}\text { Average reading, } \\
\text { (Mean, } \overline{\boldsymbol{x}}),(\mathbf{x}, \mathbf{y} \text { pixel) }\end{array}$} & $\begin{array}{l}\text { Standard } \\
\text { Deviation, } \mathbf{s}\end{array}$ \\
\hline \multicolumn{2}{|l|}{ Roberts edge detector standard deviation } \\
\hline Start & $784.4,125.3$ & $0.4,0.0$ \\
\hline Supporting 1 & $580.5,293.4$ & $0.0,0.4$ \\
\hline Supporting 2 & $775.7,462.6$ & $1.6,0.2$ \\
\hline End & $584.3,624.3$ & $0.1,1.1$ \\
\hline Sobel edge detector standard deviation \\
\hline Start & $783.4,125.3$ & $0.1,0.1$ \\
\hline Supporting 1 & $579.0,291.2$ & $0.4,0.5$ \\
\hline Supporting 2 & $777.4,464.0$ & $0.4,0.5$ \\
\hline End & $582.1,625.2$ & $0.4,0.4$ \\
\hline Prewitt edge detector standard deviation \\
\hline Start & $783.7,124.4$ & $0.1,0.0$ \\
\hline Supporting 1 & $579.1,291.4$ & $0.4,0.4$ \\
\hline Supporting 2 & $777.4,463.1$ & $0.1,0.4$ \\
\hline End & $580.3,629.3$ & $0.8,0.1$ \\
\hline Canny edge detector standard deviation \\
\hline Start & $785.8,122.4$ & $0.0,0.0$ \\
\hline Supporting 1 & $576.8,289.2$ & $0.3,0.1$ \\
\hline Supporting 2 & $778.4,463.2$ & $0.3,0.2$ \\
\hline End & $581.5,625.6$ & $0.0,0.0$ \\
\hline
\end{tabular}

Based on Table 6, it shows that all the edge detection techniques have a low value of standard deviation nearing 0 . Each reading of the coordinates of the feature points is near to its average reading, thus, low in variations. This proves that the procedure used and the determined variables for each edge detection techniques shows a high repeatability and reliability, meaning that the repeated reading on the coordinates of the features points using the same equipment, procedures, methods and variables has less spread from the average points value.

\section{CONCLUSION}

As a conclusion, the edge of a tooth saw butt joint shape has been successfully recognized and identified by using digital image processing using different edge detection techniques, morphological operation and feature points extraction, Shi-Tomasi. Canny edge detector shows the highest accuracy in detecting the feature points of the edge of a tooth saw butt joint shape due to the hysteresis threshold of the edge detector which is a two pre-determined threshold value that gives more definition to the edge detected, followed by Prewitt, Sobel and Roberts edge detector. Each of the edge detection techniques has a low value of standard deviation. It shows that, the repeated reading obtain for each of the edge detection techniques has a low variation in its readings, meaning that the methods have a high repeatability and reliability on the 
procedures and variables used.

The workpiece in this project is made from a hard cardboard material and spray painted with silver color to imitate the reflective behavior of an aluminum sheet. It is recommended for future work to use a real aluminum sheet or any mild steel to perform this project to exhibit the real characteristic behavior of those materials because the application of this project is used in industrial robotic welding. Next, for future studies, comparison can be made more by using different shape of butt joint edges, such as straight line, curved and even a higher number of sharp edges of a tooth saw shape.

\section{ACKNOWLEDGEMENT}

The authors are grateful for the support granted by by Center for Robotics and Industrial Automation, Universiti Teknikal Malaysia Melaka (UTeM) in conducting this research through grant JURNAL/2018/FKE/Q00007 and Ministry of Higher Education.

\section{REFERENCES}

1. H. N. M. Shah, M. Sulaiman, A. Z. Shukor, Z. Kamis, and A. A. Rahman, Butt welding joints recognition and location identification by using local thresholding, Robot. Comput. Integr. Manuf., vol. 51, no. December 2017, pp. 181-188, 2018.

2. M. Dinham and G. Fang, Autonomous weld seam identification and localisation using eye-in-hand stereo vision for robotic arc welding, Robot. Comput. Integr. Manuf., vol. 29, no. 5, pp. 288-301, 2013.

3. Y. He, Y. Xu, Y. Chen, H. Chen, and S. Chen, Weld seam profile detection and feature point extraction for multi-pass route planning based on visual attention model, Robot. Comput. Integr. Manuf., vol. 37, pp. 251-261, 2016.

4. D. Kaur and Y. Kaur, Various Image Segmentation Techniques: A Review, Int. J. Comput. Sci. Mob. Comput., vol. 3, no. 5, p. 809-814, date accessed: 18/05/2016, 2014.

5. K. Bala Krishnan, S. Prakash Ranga, and N. Guptha, A Survey on Different Edge Detection Techniques for Image Segmentation, Indian J. Sci. Technol., vol. 10, no. 4, 2017.

6. Y. Zou, Y. Wang, W. Zhou, and X. Chen, Real-time seam tracking control system based on line laser visions, Opt. Laser Technol., vol. 103, pp. 182-192, 2018.

7. Y. Xu., Lv, N., Fang, G., Du, S., Zhao, W., Ye, Z. and Chen, S., Welding seam tracking in robotic gas metal arc welding, J. Mater. Process. Technol., vol. 248, no. May, pp. 18-30, 2017. https://doi.org/10.1016/j.jmatprotec.2017.04.025

8. W. J. Shao, Y. Huang, and Y. Zhang, A novel weld seam detection method for space weld seam of narrow butt joint in laser welding, Opt. Laser Technol., vol. 99, pp. 39-51, 2018.

9. Y. Zou, X. Chen, G. Gong, and J. Li, A seam tracking system based on a laser vision sensor, Meas. J. Int. Meas. Confed., vol. 127, no. February, pp. 489-500, 2018.

10. J. Fan, F. Jing, L. Yang, T. Long, and M. Tan, A precise seam tracking method for narrow butt seams based on structured light vision sensor, Opt. Laser Technol., vol. 109, no. 95, pp. 616-626, 2019.

11. J. Zeng, B. Chang, D. Du, Y. Hong, Y. Zou, and S. Chang, A visual weld edge recognition method based on light and shadow feature construction using directional lighting, J. Manuf. Process., vol. 24, pp. 19-30, 2016.

12. H. N. M. Shah, M. Sulaiman, A. Z. Shukor, and M. Z. A. Rashid, Vision Based Identification and Detection of Initial , Mid and End Points of Weld Seams Path in ButtWelding Joint using Point Detector Methods, vol. 8, no. 7, pp. 57-61, 1843.

13. Ghandi, N. (2018, July 24). Harris Corner Detection and Shi-Tomasi Corner Detection [Online]. Available: https://medium.com/pixel-wise/detect-those-corners-aba 0f034078b

14. B. G. Batchelor and F. M. Waltz, Morphological image processing, Mach. Vis. Handb., vol. 8491, pp. 802-870, 2012. https://doi.org/10.1007/978-1-84996-169-1_19

15. S. Javier and N. Monz, An Analysis and Implementation of the Harris Corner Detector The Harris Corner Detector, vol. 8, pp. 305-328, 2018.

16. H. N. M. Shah, M. Sulaiman, A. Z. Shukor, and Z. Kamis, Recognition and identification the position and location of tooth saw butt joint shape, Int. J. Adv. Manuf. Technol., vol. 98, no. 9-12, pp. 2497-2504, 2018.

17. Jeyalaksshmi, S., Prasanna, S. Image Segmentation Using Various Edge Detection Techniques, International Journal of Emerging Technologies in Engineering Research (IJETER), vol. 4, no. 7, pp. 129-132, 2016.

18. Hema, D., Pavan Kumar, K., Harish Devarayal, M., Bharath, G., Harish, N.S., Object Detection Using Image Processing With LTP Features, International Journal of Emerging Technologies in Engineering Research (IJETER), vol. 6, no.4, 2018. 